OPEN ACCESS

Edited by:

Xianming Ye,

University of Pretoria, South Africa

Reviewed by

Bo Wang,

Huazhong University of Science and

Technology, China

Zhou Wu,

Chongqing University, China

*Correspondence: Bing Zhu

zhubing@buaa.edu.cn

Specialty section:

This article was submitted to Process and Energy Systems

Engineering,

a section of the journal Frontiers in Energy Research

Received: 31 August 2021 Accepted: 11 October 2021

Published: 18 November 2021

Citation:

Zheng $W$ and Zhu B (2021) Stochastic Time-Varying Model Predictive Control for Trajectory Tracking of a Wheeled

Mobile Robot.

Front. Energy Res. 9:767597.

doi: 10.3389/fenrg.2021.767597

\section{Stochastic Time-Varying Model Predictive Control for Trajectory Tracking of a Wheeled Mobile Robot}

\author{
Weijiang Zheng and Bing Zhu* \\ The Seventh Research Division, Beihang University, Beijing, China
}

In this paper, a stochastic model predictive control (MPC) is proposed for the wheeled mobile robot to track a reference trajectory within a finite task horizon. The wheeled mobile robot is supposed to subject to additive stochastic disturbance with known probability distribution. It is also supposed that the mobile robot is subject to soft probability constraints on states and control inputs. The nonlinear mobile robot model is linearized and discretized into a discrete linear time-varying model, such that the linear time-varying MPC can be applied to forecast and control its future behavior. In the proposed stochastic MPC, the cost function is designed to penalize its tracking error and energy consumption. Based on quantile techniques, a learning-based approach is applied to transform the probability constraints to deterministic constraints, and to calculate the terminal constraint to guarantee recursive feasibility. It is proved that, with the proposed stochastic MPC, the tracking error of the closed-loop system is asymptotically average bounded. A simulation example is provided to support the theoretical result.

Keywords: model predictive control, mobile robot, probability constraint, linear time-varying systems, optimization

\section{INTRODUCTION}

Model predictive control (MPC) is a useful tool when dealing with stabilization or tracking problem with constraints. It solves a finite horizon optimal control problem in a receding horizon manner while only implementing one-step-ahead predictive control at next time step. please see the survey paper Mayne et al. (2000) for more detailed directions of MPC. Some recent results on MPC include (but do not limited to) adaptive MPC (Zhu et al., 2020), distributed MPC (Zhu et al., 2018; Wang et al., 2020), robust MPC (Yang et al., 2021), and stochastic MPC (Hewing and Zeilinger, 2018). Due to its advantages on improving system performance and handling constraints, MPC can be applied to motion control (Alcalá et al., 2020), process control (Wu et al., 2020), energy systems (Stadler et al., 2018; Rodas et al., 2021), etc. Several approaches of Robust MPC for wheeled mobile robots have been studied in Gonzalez et al. (2011) and Sun et al. (2018). However, conservative results will be obtained owing to disturbances (Mayne, 2016).

Stochastic MPC resolves the contradiction of safety and economy by taking advantage of uncertainties with known distributions. In addition, violations of constraints are permitted in a predetermined level and thus the soft constraints can balance system performance and limits on states in the meantime. Such characteristic renders stochastic MPC a promising method in a wide range of applications in energy or under actuated systems (Farina et al., 2016), such as energy scheduling (Rahmani-andebili and Shen, 2017), (Scarabaggio et al., 2021), (Jørgensen et al., 2016), energy management for vehicles (Cairano et al., 2014), temperature control in buildings (Hewing and Zeilinger, 2018), racing car Carrau et al. (2016), quadrotors (Yang et al., 2017), overhead cranes 


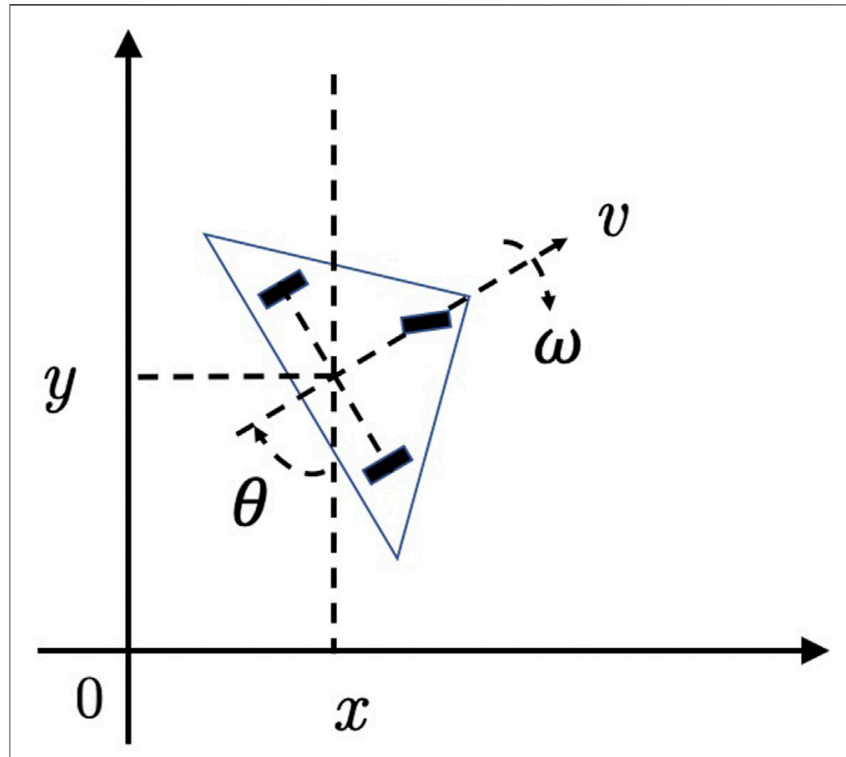

FIGURE 1 | Fundamental structure of the wheeled mobile robot.

(Wu et al., 2015), automated driving vehicles (Suh et al., 2018) and mobile robots (Goncalves et al., 2018). The majority of approaches in Stochastic MPC community are developed for LTI systems (Mesbah, 2016). Several attempts in motion control have been made for LTV or nonlinear systems with ignoring requirement of recursive feasibility (Yang et al., 2017; Goncalves et al., 2018).

This paper presents a stochastic MPC method for wheeled mobile robots based on framework of Hewing et al. (2020). The proposed method remains valid for other LTV systems or nonlinear systems after linearization. The main contribution is to develop a stochastic MPC method to forecast and control the wheeled mobile robot to track its reference trajectory against constraints and additive random disturbance with known distributions. In the proposed stochastic MPC, the cost function is designed to penalize both the tracking error and the energy consumption. The learning-based technique and probabilistic reachable set are applied, such that probability constraints can be transformed to deterministic constraints, and the terminal constraint can be calculated to ensure the recursive feasibility and asymptotic average boundedness. A simulation example with its simulated statistical data is provided to validate the proposed theoretical results.

The rest of the paper is arranged as follows. In Section 2, the LTV kinematic model for the mobile robot is introduced, and the control problem is formulated. In Section 3, the proposed stochastic MPC is designed in detail. In Section 4, recursive feasibility of optimization and asymptotic average boundedness are analysed in detail. In Section 5, the simulation result is provided to show the efficacy of the method.

\section{PROBLEM STATEMENT}

The wheeled mobile robot consists of two differential driving wheels and a guide wheel, as illustrated by Figure 1. Translational velocity and angular velocity of the robot can be expressed by rotation rates of driving wheels as $v=\frac{r\left(\omega_{\text {right }}+\omega_{\text {left }}\right)}{2}$ and $\omega=\frac{r\left(\omega_{\text {right }}-\omega_{\text {left }}\right)}{l}$, where $r$ and $l$ denote the radius and the distance of/between driving wheels, respectively; $\omega_{\text {left }}$ and $\omega_{\text {right }}$ are the rotational rates of the left and right wheels, respectively. Choosing generalized coordinate as state, velocity as control and taking additive disturbance into consideration, the kinematic model is described by

$$
\dot{\mathbf{q}}=\left[\begin{array}{c}
\dot{x} \\
\dot{y} \\
\dot{\theta}
\end{array}\right]=\left[\begin{array}{cc}
\cos \theta & 0 \\
\sin \theta & 0 \\
0 & 1
\end{array}\right]\left[\begin{array}{c}
v \\
\omega
\end{array}\right]+\left[\begin{array}{l}
w_{1} \\
w_{2} \\
w_{3}
\end{array}\right]=f(\mathbf{q}, \mathbf{u})+\mathbf{w}
$$

where $\mathbf{q}=[x, y, \theta]^{T}$ denotes the position and orientation of the mobile robot; $u=[v, \omega]^{T}$ denotes the translational velocity and angular velocity, which are considered as the inputs of the system; $\left.\mathbf{w}=\left[w_{1}, w_{2}, w_{3}\right)\right]^{T}$ is the disturbance with bounded or unbounded support and is subject to a known probability distribution under zero-mean assumption (Guassian distribution, e.g.).

The reference model to be tracked is given by

$$
\dot{\mathbf{q}}_{r}=\left[\begin{array}{c}
\dot{x}_{r} \\
\dot{y}_{r} \\
\dot{\theta}_{r}
\end{array}\right]=\left[\begin{array}{cc}
\cos \theta_{r} & 0 \\
\sin \theta_{r} & 0 \\
0 & 1
\end{array}\right]\left[\begin{array}{c}
v_{r} \\
\omega_{r}
\end{array}\right]=f\left(\mathbf{q}_{r}, \mathbf{u}_{r}\right),
$$

where $x_{r}, y_{r}, \theta_{r}$ are the reference position and orientation, respectively.

Define the tracking error by $\tilde{\mathbf{q}}=\mathbf{q}-\mathbf{q}_{r}$. The dynamics of the tracking error can be linearized by

$$
\dot{\tilde{\mathbf{q}}}=A_{c}(t) \tilde{\mathbf{q}}+B_{c}(t) \tilde{\mathbf{u}}+\mathbf{w},
$$

where $\tilde{\mathbf{u}}=\mathbf{u}-\mathbf{u}_{r} ; A_{c}(t)$ and $B_{c}(t)$ are time-varying matrices calculated by

$$
A_{c}(t)=\left.\frac{\partial f}{\partial \mathbf{q}}\right|_{\mathbf{q}=\mathbf{q}_{r}}, \quad B_{c}(t)=\left.\frac{\partial f}{\partial \mathbf{u}}\right|_{\mathbf{u}=\mathbf{u}_{r}} .
$$

If the sampling interval $T$ is chosen small enough, it is appropriate to apply Euler discretization to obtain the timevarying linear discrete-time model:

$$
\tilde{\mathbf{q}}(k+1)=A(k) \tilde{\mathbf{q}}(k)+B(k) \tilde{\mathbf{u}}(k)+T \mathbf{w}(k)
$$

where $T$ is the sampling period; $k$ is the sampling time; and

$$
\begin{aligned}
& A(k)=\left[\begin{array}{ccc}
1 & 0 & -v_{r}(k) \sin \theta_{r}(k) T \\
0 & 1 & v_{r}(k) \cos \theta_{r}(k) T \\
0 & 0 & 1
\end{array}\right] \\
& B(k)=\left[\begin{array}{cc}
\cos \theta_{r}(k) T & 0 \\
\sin \theta_{r}(k) T & 0 \\
0 & T
\end{array}\right]
\end{aligned}
$$

It is supposed that the mobile robot is subject to the probability constraints:

$$
\begin{aligned}
& P\left\{\mathbf{V}_{q} \tilde{\mathbf{q}}(k) \leq \mathbf{1}\right\} \geq \mathbf{p}_{x}, \\
& P\left\{\mathbf{V}_{u} \tilde{\mathbf{u}}(k) \leq \mathbf{1}\right\} \geq \mathbf{p}_{u},
\end{aligned}
$$

where $\mathbf{V}_{q}$ and $\mathbf{V}_{u}$ are constant matrices with proper dimensions; $\mathbf{p}_{x}$ and $\mathbf{p}_{u}$ are constant vectors with proper dimensions. It can be 
seen from (6) and $\mathbf{7}$ that, if $\mathbf{p}_{x}=\mathbf{1}$ or $\mathbf{p}_{u}=\mathbf{1}$, then the constraints appear to be deterministic.

The objective of this paper is that, design a model predictive controller, such that the mobile robot (subject to stochastic disturbance) is capable of tracking its reference trajectory within a finite task horizon $\bar{N}>0$, or equivalently, its tracking error $\tilde{\mathbf{q}}$ is subject to zero mean and bounded covariance within a finite task horizon.

\section{STOCHASTIC LINEAR TIME-VARYING MPC}

In this section, the robust dual-mode MPC is applied as the fundamental structure. At time $k$, the predictive control are set by.

$$
\begin{aligned}
& \tilde{\mathbf{u}}(i \mid k)=K(k+i) \mathbf{e}(i \mid k)+\mathbf{v}(i \mid k), \quad i=0, \ldots, N-1, \\
& \tilde{\mathbf{u}}(i \mid k)=K(k+i) \mathbf{e}(i \mid k)+K(k+i) \mathbf{s}(i \mid k), \quad i \geq N,
\end{aligned}
$$

where the notation $(\cdot)(i \mid k)$ denotes the $i$ th step prediction at time $k$; $N$ is the predictive horizon. In this paper, the control horizon is set equal to the predictive horizon. The tracking error $\tilde{\mathbf{q}}$ is decomposed into the nominal state $\mathbf{s}$ and the error e, namely.

$$
\begin{gathered}
\tilde{\mathbf{q}}(i \mid k)=\mathbf{s}(i \mid k)+\mathbf{e}(i \mid k), \\
\mathbf{s}(i+1 \mid k)=A(k+i) \mathbf{s}(i \mid k)+B(k+i) \mathbf{v}(i \mid k), \\
\mathbf{e}(i+1 \mid k)=\Phi(k+i) \mathbf{e}(i \mid k)+T \mathbf{w}(i \mid k), \\
\Phi=A+B K .
\end{gathered}
$$

where $\mathbf{v}$ is the nominal MPC for the nominal state $\mathbf{s}$ K is obtained from solving the following Riccati equation at every time instant:

$$
\begin{gathered}
S=A^{T} S A+Q-A^{T} S B\left(B^{T} S B+R\right)^{-1} B^{T} S A, \\
K=-\left(B^{T} S B+R\right)^{-1} B^{T} S A .
\end{gathered}
$$

where $Q$ and $R$ are the positive-definite weighting matrices in MPC design; $S$ is to be solved based on the given $Q$ and $R$. In (14) and $\mathbf{1 5}$, we have dropped $k$ for brevity.

\subsection{Cost Function}

The cost function in MPC is designed by

$$
J(k)=\mathbb{E}\left[\sum_{j=0}^{N-1}\left(\|\tilde{\mathbf{q}}(j \mid k)\|_{Q}^{2}+\|\tilde{\mathbf{u}}(j \mid k)\|_{R}^{2}\right)+\|\tilde{\mathbf{q}}(N \mid k)\|_{P(k)}^{2}\right],
$$

where $Q$ and $R$ are the positive-definite weighting matrices, and $p(k)$ is solved from the following Lyapunov equation at every time step $k-1$ :

$$
\begin{aligned}
P(k+1)=Q & +K^{T}(k+N) R K(k+N) \\
& +\Phi^{T}(k+N) P(k+1) \Phi(k+N) .
\end{aligned}
$$

In (16), the first term is to penalize the tracking error; the second term is to penalize the control power (which reflects the energy efficiency); and the third term is the terminal cost to ensure the overall feasibility and average performance.

The forecasting equation of the nominal part of the system dynamics can be rewritten in the compact form:

$$
\overline{\mathbf{s}}(k+1)=\bar{A}(k) \mathbf{s}(0 \mid k)+\bar{B}(k) \overline{\mathbf{v}}(k),
$$

where.

$$
\begin{gathered}
\overline{\mathbf{s}}(k+1)=[\mathbf{s}(1 \mid k), \ldots, \mathbf{s}(N \mid k)]^{T}, \\
\overline{\mathbf{v}}(k)=[\mathbf{v}(0 \mid k), \ldots, \mathbf{v}(N-1 \mid k)]^{T}, \\
A(k)=\left[\begin{array}{c}
A(0 \mid k) \\
A(1 \mid k) A(0 \mid k) \\
\vdots \\
\prod_{i=1}^{N-1} A(i \mid k)
\end{array}\right], \\
B(0 \mid k) \\
\bar{B}(k)=\left[\begin{array}{c}
A(1 \mid k) B(0 \mid k) \\
\vdots \\
\prod_{i=1}^{N-1} A(i \mid k) B(0 \mid k) \prod_{i=2}^{N-1} A(i \mid k) B(1 \mid k) \cdots B(N-1 \mid k)
\end{array}\right] .
\end{gathered}
$$

Then the cost function can be written by

$$
\begin{aligned}
J(k)= & \|\overline{\mathbf{s}}(k+1)\|_{\bar{Q}}^{2}+\|\overline{\mathbf{v}}(k)\|_{\bar{R}}^{2}+\|\mathbf{s}(0 \mid k)\|_{Q}^{2} \\
& +\mathbb{E}\left[\sum_{j=0}^{N-1}\left(\|\mathbf{e}(j \mid k)\|_{Q+K^{T}(k) R K(k)}^{2}\right)+\|\mathbf{e}(N \mid k)\|_{P(k)}^{2}\right]
\end{aligned}
$$

where

$$
\begin{aligned}
\bar{Q} & =\operatorname{diag}[Q, Q, \ldots, Q, P(k)], \\
\bar{R} & =\operatorname{diag}[R, R, \ldots, R] .
\end{aligned}
$$

The cost function can be further rewritten by

$$
J(k)=\frac{1}{2}\|\overline{\mathbf{v}}(k)\|_{H}^{2}+\mathbf{f}^{T}(k) \overline{\mathbf{v}}(k)+d(k)
$$

where

$$
\begin{aligned}
& H=2\left(\bar{B}^{T}(k) \bar{Q} \bar{B}(k)+\bar{R}\right), \\
& \mathbf{f}=2 \bar{B}^{T}(k) \bar{Q}^{T} \bar{A}(k) \mathbf{s}(0 \mid k),
\end{aligned}
$$

and

$$
\begin{aligned}
d(k)= & \|\mathbf{s}(0 \mid k)\|_{Q+\bar{A}^{T}(k) \bar{Q}^{T} \bar{A}(k)}^{2} \\
& +\mathbb{E}\left[\sum_{j=0}^{N-1}\left(\|\mathbf{e}(j \mid k)\|_{Q+K^{T}(k) R K(k)}^{2}\right)+\|\mathbf{e}(N \mid k)\|_{P(k)}^{2}\right] .
\end{aligned}
$$

It can be seen that $d(k)$ is independent of $\mathbf{v}(k)$.

\subsection{Constraints}

In MPC design, the constraints (6) and (7) have to be handled. Let

$$
\begin{aligned}
& X=\left\{\tilde{\mathbf{q}} \mid \mathbf{V}_{q} \tilde{\mathbf{q}}(k) \leq \mathbf{1}\right\}, \\
& U=\left\{\tilde{\mathbf{u}} \mid \mathbf{V}_{u} \tilde{\mathbf{u}}(k) \leq \mathbf{1}\right\} .
\end{aligned}
$$

Here, we apply the technique of probabilitic reacheable set (Hewing and Zeilinger, 2018). The k-step probabilistic reachable sets can be calculated by.

$$
\begin{aligned}
& R_{k, x}=\left\{\mathbf{e} \mid \mathbf{V}_{q} \mathbf{e}(k) \leq \gamma_{x}(k)\right\}, \\
& R_{k, u}=\left\{\mathbf{e} \mid \mathbf{V}_{u} \mathbf{e}(k) \leq \gamma_{u}(k)\right\} .
\end{aligned}
$$


where

$$
\begin{gathered}
P\left\{\mathbf{V}_{q} \mathbf{e}(k) \leq \gamma_{x}(k)\right\} \geq \mathbf{p}_{x}, \\
P\left\{\mathbf{V}_{u} K(k) \mathbf{e}(k) \leq \gamma_{u}(k)\right\} \geq \mathbf{p}_{u},
\end{gathered}
$$

for $i=1,2, \ldots, \bar{N}$, and $\bar{N} \gg N$. Several methods for computing the quantiles $\gamma_{x}(k)$ and $\gamma_{u}(k)$ are summarized in Farina et al. (2016). One numerical approximation is to discretize the distributions of w, e.g. Kouvaritakis et al. (2010), while another adopts the framework of scenario MPC.

The distribution of $\mathbf{e}(k)$ can be computed with distributions of $\mathbf{w}(k)$ by the predictive error dynamics

$$
\left[\begin{array}{c}
\mathbf{e}(1) \\
\mathbf{e}(2) \\
\vdots \\
\mathbf{e}(\bar{N})
\end{array}\right]=T\left[\begin{array}{cccc}
I & 0 & \cdots & 0 \\
\Phi(1) & I & \ddots & \vdots \\
\vdots & \vdots & \ddots & 0 \\
\bar{N}-1 & \bar{N}-1 & \\
\prod_{i=1} \Phi(i) & \prod_{i=2} \Phi(i) & \cdots & I
\end{array}\right]\left[\begin{array}{c}
\mathbf{w}(0) \\
\mathbf{w}(1) \\
\vdots \\
\mathbf{w}(\bar{N}-1)
\end{array}\right] .
$$

Constraints for nominal states $\mathbf{s}(k)$ can be calculated by

$$
S_{k}=X \odot R_{k, x},
$$

or specifically

$$
\left(S_{k}\right)_{j}=\left\{\mathbf{s} \mid\left(V_{q}\right)_{j} \mathbf{s} \leq 1-\max _{\mathbf{e} \in R_{k, x}}\left(V_{q}\right)_{j} \mathbf{e}\right\}
$$

where $j=1, \ldots, n_{V}$ denotes the $j$ th row of the matrix. The constraint (37) can be rewritten in compact form by

$$
S_{k}=\left\{\mathbf{s} \mid V_{q} \mathbf{s} \leq \mathbf{s}_{k}\right\} .
$$

The constraints for nominal inputs $\mathbf{v}(k)$ can be calculated in the similar process, namely,

$$
V_{k}=U \odot K R_{k, u},
$$

where

$$
K R_{k, u}=\left\{K(k) \mathbf{e} \mid \mathbf{e} \in R_{k, u}\right\}, V_{k}=\left\{\mathbf{v} \mid V_{u} \mathbf{v} \leq \mathbf{v}_{k}\right\},
$$

or specifically

$$
\left(V_{k}\right)_{j}=\left\{\mathbf{v} \mid\left(V_{u}\right)_{j} \mathbf{v} \leq 1-\max _{\mathbf{e} \in R_{k, u}}\left(V_{u}\right)_{j} K(k) \mathbf{e}\right\} .
$$

For the recursive feasibility of optimization in MPC algorithm, terminal state constraints are imposed on the nominal states:

$$
\mathbf{s}(N \mid k) \in S_{T}
$$

where $S_{T}$ is a positive invariant set for the nominal system.

The terminal state set $S_{T}$ can be defined based on the terminal control set:

$$
\begin{gathered}
V_{T} \subseteq V_{\text {inf }}=\bigcap_{k=1}^{\bar{N}} V_{k}=\left\{\mathbf{v} \mid V_{u} \mathbf{v} \leq \mathbf{v}_{\text {inf }}\right\}, \\
S_{T}=\left\{\mathbf{s} \mid \mathbf{v}=K(k) \mathbf{s} \in V_{\text {inf }}, A(k) \mathbf{s}+B(k) \mathbf{v} \in S_{T}, k=1,2, \ldots, \bar{N}\right\} .
\end{gathered}
$$

where $\mathbf{v}_{\text {inf }}$ is the smallest $\mathbf{v}_{k}$ in (40).
An iterative learning-based procedure (Gonzalez et al., 2011) can be applied to calculate the terminal constraint:

$$
Z(i+1)=\left\{\mathbf{s} \mid K(k) \mathbf{s} \in V_{i n f}, \Phi(k) \mathbf{s} \in Z(i), k=1,2, \ldots, \bar{N}\right\},
$$

where the sets are nested, i.e. $Z(i) \subseteq Z(i-1) \subseteq \cdots \subseteq Z(0)$, and

$$
Z(0)=\left\{\mathbf{s} \mid \mathbf{s} \in S_{\text {inf }}, K(k) \mathbf{s} \in V_{\text {inf }}\right\},
$$

where $S_{i n f}=\bigcap_{k=1}^{\bar{N}} S_{k}=\left\{\mathbf{s} \mid V_{q} \mathbf{s} \leq \mathbf{s}_{i n f}\right\}$, and $\mathbf{s}_{i n f}$ is the smallest $\mathbf{s}_{k}$ in (38).

The calculation of $Z(1)$ continues with $Z(i+1)=Z(1) \cap Q(i)$, where

$$
Q(i)=\stackrel{N}{N}_{k=1}^{\bar{N}} Q_{k}(Z(i)), \quad Q_{k}(Z(i))=\{\mathbf{s} \mid \Phi(k) \mathbf{s} \in Z(i)\} .
$$

Then the terminal set can be approximated by $S_{T}=Z(1)$ when $Z(i+1)=Z(i)$.

\subsection{Optimization and Implementation}

Based on the above cost function and constraints, the optimization in each time instant of MPC can be constructed by

$$
\overline{\mathbf{v}}^{*}=\arg \min _{\overline{\mathbf{v}}} J(k),
$$

subject to.

$$
\begin{aligned}
& \mathbf{s}(i \mid k) \in S_{k+i}, i=1,2, \ldots, N-1, \\
& \mathbf{v}(i \mid k) \in V_{k+i}, i=0,1, \ldots, N-1, \\
& \mathbf{s}(N \mid k) \in S_{T} .
\end{aligned}
$$

The nominal MPC can be implemented by receding horizon scheme:

$$
\mathbf{v}(k)=\left[I_{2 \times 2}, 0, \ldots, 0\right] \overline{\mathbf{v}}^{*}(k),
$$

and the stochastic MPC for the mobile robot can be implemented by

$$
\mathbf{u}(k)=\mathbf{v}(k)+K(k) \mathbf{e}(k) .
$$

\section{ANALYSIS ON THE CLOSED-LOOP SYSTEM}

\subsection{Feasibility of the Optimization}

In this paper, the recursive feasibility is slightly different from the conventional feasibility of MPC. The aim is to prove that the optimization 48) is recursively feasible throughout the task horizon $\bar{N}$. Specifically, it is to prove that, if the optimization 48) is feasible at $k=0$, then it is feasible at $k=1,2, \ldots, \bar{N}-N$, where $\bar{N} \gg N$.

To prove the recursive feasibility within the task horizon, the "tail" method can be applied here. Suppose that the optimization is feasible at time $k$. At time $k+1$, a feasible solution can be constructed by using the "tail" of the optimal solution at time $k$, namely, 
$\tilde{\mathbf{v}}(k+1)=\left[\mathbf{v}^{T}(1 \mid k), \mathbf{v}^{T}(2 \mid k), \ldots, \mathbf{v}^{T}(N-1 \mid k),(K(k+N) \mathbf{s}(N \mid k))^{T}\right]^{T}$,

which satisfies the input constraint (50). Moreover, in (54), $K(k+$ $N) \mathbf{s}(N \mid k) \in V_{\text {inf }} \subseteq V_{k+N}$, indicating that the terminal constraint (51) is satisfied. Satisfaction of state constraint (49) can also be checked in a similar way.

Satisfaction of constraints (49)-(51) indicates that the original stochastic constraints (6) and (7) are satisfied. Consequently, at least one feasible solution exists at time $k+1$, if the optimization is feasible at time $k$. The optimization in (48) is recursively feasible.

\subsection{Average Asymptotic Boundedness of the Closed-Loop System}

In this section, the conventional concept of asymptotic stability is inapplicable, since only performances within the task horizon are concerned. We are aiming to prove that the states and inputs satisfy all constraints, while the states are average asymptotically bounded.

Let $J^{\star}(k)$ denote the optimal cost at time step $k$. Let $J^{-}(k)$ denote the feasible cost at time step $k$ by using the "tail" information at time $k-1$. Specifically,

$$
J^{*}(k)=\mathbb{E}\left[\sum_{j=0}^{N-1}\left(\left\|\tilde{\mathbf{q}}^{*}(j \mid k)\right\|_{Q}^{2}+\left\|\tilde{\mathbf{u}}^{*}(j \mid k)\right\|_{R}^{2}\right)+\left\|\tilde{\mathbf{q}}^{*}(N \mid k)\right\|_{P(k)}^{2}\right],
$$

where ${ }^{*}$ denotes the optimal control or state sequence. And

$$
\begin{aligned}
J^{-}(k+1)= & \mathbb{E}\left[\sum_{j=1}^{N-1}\left(\left\|\tilde{\mathbf{q}}^{\star}(j \mid k)\right\|_{Q}^{2}+\left\|\tilde{\mathbf{u}}^{*}(j \mid k)\right\|_{R}^{2}\right)+\left\|\tilde{\mathbf{q}}^{*}(N \mid k)\right\|_{Q}^{2}\right. \\
& \left.+\left\|K(k+N) \tilde{\mathbf{q}}^{*}(N \mid k)\right\|_{R}^{2}\right] \\
& +\mathbb{E}\left[\left\|\Phi(k+N) \tilde{\mathbf{q}}^{*}(N \mid k)+T \mathbf{w}(N \mid k)\right\|_{P(k+1)}^{2}\right]
\end{aligned}
$$

It follows that

$J^{*}(k+1)-J^{*}(k) \leq J^{-}(k+1)-J^{*}(k)$

$=\mathbb{E}\left[\left\|\tilde{\mathbf{q}}^{*}(N \mid k)\right\|_{Q}^{2}-\left\|\tilde{\mathbf{q}}^{*}(0 \mid k)\right\|_{Q}^{2}+\left\|K(k+N) \tilde{\mathbf{q}}^{*}(N \mid k)\right\|_{R}^{2}-\left\|\tilde{\mathbf{u}}^{*}(0 \mid k)\right\|_{R}^{2}\right]$

$+\mathbb{E}\left[\left\|\Phi(k+N) \tilde{\mathbf{q}}^{*}(N \mid k)+T \mathbf{w}(N \mid k)\right\|_{P(k+1)}^{2}-\left\|\tilde{\mathbf{q}}^{*}(N \mid k)\right\|_{P(k)}^{2}\right]$

$=\mathbb{E}\left[\left\|\tilde{\mathbf{q}}^{*}(N \mid k)\right\|_{Q+K^{T}(k+N) R K(k+N)+\Phi^{T}(k+N) P(k+1) \Phi(k+N)-P(k)}^{2}\right]$

$+\mathbb{E}\left[\|T \mathbf{w}(N \mid k)\|_{P(k+1)}^{2}-\left\|\tilde{\mathbf{q}}^{*}(0 \mid k)\right\|_{Q}^{2}-\left\|\tilde{\mathbf{u}}^{*}(0 \mid k)\right\|_{R}^{2}\right]$.

It then follows from (17) that

$$
\begin{aligned}
& J^{*}(k+1)-J^{*}(k) \leq-\left\|\tilde{\mathbf{q}}^{*}(k)\right\|_{Q}^{2}-\left\|\tilde{\mathbf{u}}^{*}(k)\right\|_{R}^{2} \\
& \quad+\mathbb{E}\left[\left\|\tilde{\mathbf{q}}^{*}(N \mid k)\right\|_{P(k+1)-P(k)}^{2}+\|T \mathbf{w}(N \mid k)\|_{P(k+1)}^{2}\right] .
\end{aligned}
$$

Summing both sides of the above inequality for $k=0,1,2 \ldots \bar{N}-N-1$, and dividing the result by $\bar{N}-N$ yields that

$$
\begin{aligned}
& \frac{1}{\bar{N}-N}\left(J^{*}(\bar{N}-N)-J^{*}(0)\right)+\frac{1}{\bar{N}-N} \sum_{k=0}^{\bar{N}-N}\left(\left\|\tilde{\mathbf{q}}^{*}(k)\right\|_{Q}^{2}+\left\|\tilde{\mathbf{u}}^{*}(k)\right\|_{R}^{2}\right) \\
& \leq \frac{1}{\bar{N}-N} \sum_{k=0}^{\bar{N}-N} \mathbb{E}\left[\left\|\tilde{\mathbf{q}}^{*}(N \mid k)\right\|_{P(k+1)-P(k)}^{2}+\|T \mathbf{w}(N \mid k)\|_{P(k+1)}^{2}\right],
\end{aligned}
$$

where $J^{*}(0)$ is finite, and $J^{*}(\bar{N}-N)$ is finite based on recursive feasibility. It then follows that

$$
\lim _{\bar{N} \rightarrow+\infty} \frac{1}{\bar{N}-N}\left(\sum_{k=0}^{\bar{N}-N}\left(\left\|\tilde{\mathbf{q}}^{*}(k)\right\|_{Q}^{2}+\left\|\tilde{\mathbf{u}}^{*}(k)\right\|_{R}^{2}\right)\right) \leq C+\mathbb{E}\left[\|T \mathbf{w}\|_{P_{2}}^{2}\right],
$$

where

$$
\begin{aligned}
& P_{2}=\operatorname{diag}\left(\max _{k}\left(P_{11}(k+1)\right), \max _{k}\left(P_{22}(k+1)\right), \max _{k}\left(P_{33}(k+1)\right)\right), \\
& C=\max _{k}\left(\mathbb{E}\left[\left\|\tilde{\mathbf{q}}^{*}(N \mid k)\right\|_{P(k+1)-P(k)}^{2}\right]\right) .
\end{aligned}
$$

The limitation 56) indicates that the tracking error of the closed-loop system is asymptotic average bounded.

\section{SIMULATION}

A simulation example is presented to validate the performance of the proposed method. In simulation, the sampling interval is $T=0.1 \mathrm{~s}$; and the task horizon is given by 13s. Initial conditions of the mobile robot and the reference model are supposed to be $q(0)=$ $[0.05,-0.02,0.05)]^{T}$ and $q_{r}(0)=[0,0,0]^{T}$, respectively. It is suppose that there exists additive disturbances subject to a normal distribution: $\mathbf{w} \sim \mathcal{N}(0, \Sigma)$, where $\Sigma=\operatorname{diag}\left(0.4^{2}, 0.3^{2}, 0.1^{2}\right)$. The reference trajectory for the mobile robot to track is a circular:

$$
x_{r}(k)=\frac{12}{\pi} \sin \left(\frac{\pi}{6} k\right), \quad y_{r}(k)=\frac{12}{\pi} \cos \left(\frac{\pi}{6} k-\pi\right)+\frac{12}{\pi} .
$$

The control inputs are subject to the following constraints:

$$
-1 \mathrm{~m} / \mathrm{s} \leq \tilde{v} \leq 1 \mathrm{~m} / \mathrm{s}, \quad-\frac{\pi}{2} \mathrm{rad} / \mathrm{s} \leq \tilde{\omega} \leq \frac{\pi}{2} \mathrm{rad} / \mathrm{s}
$$

where $\tilde{v} \triangleq v-v_{r}$ and $\tilde{\omega} \triangleq \omega-\omega_{r}$, and $v_{r}$ and $\omega_{r}$ can be calculated from (57) and 2. Here, we allow constraints (58) be violated with very small probability, i.e., they are subject to probability constraints given by (7), where

$$
V_{u}=\left[\begin{array}{cc}
1 & 0 \\
0 & \frac{2}{\pi} \\
-1 & 0 \\
0 & -\frac{2}{\pi}
\end{array}\right], \quad p_{u}=\left[\begin{array}{l}
0.99 \\
0.99 \\
0.99 \\
0.99
\end{array}\right] .
$$

The probability constraints on states are supposed to be given by (6), where 


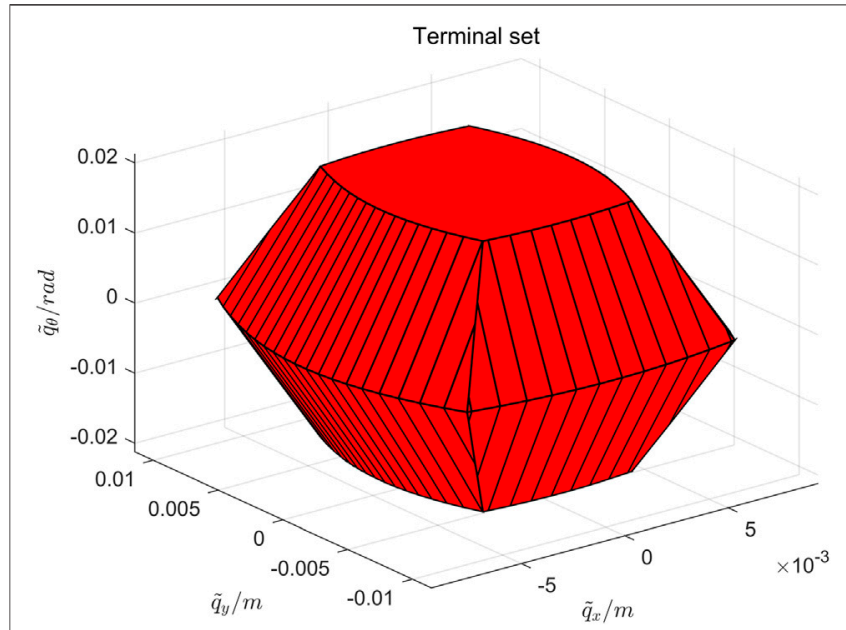

FIGURE 2 | The terminal set in the proposed stochastic MPC.

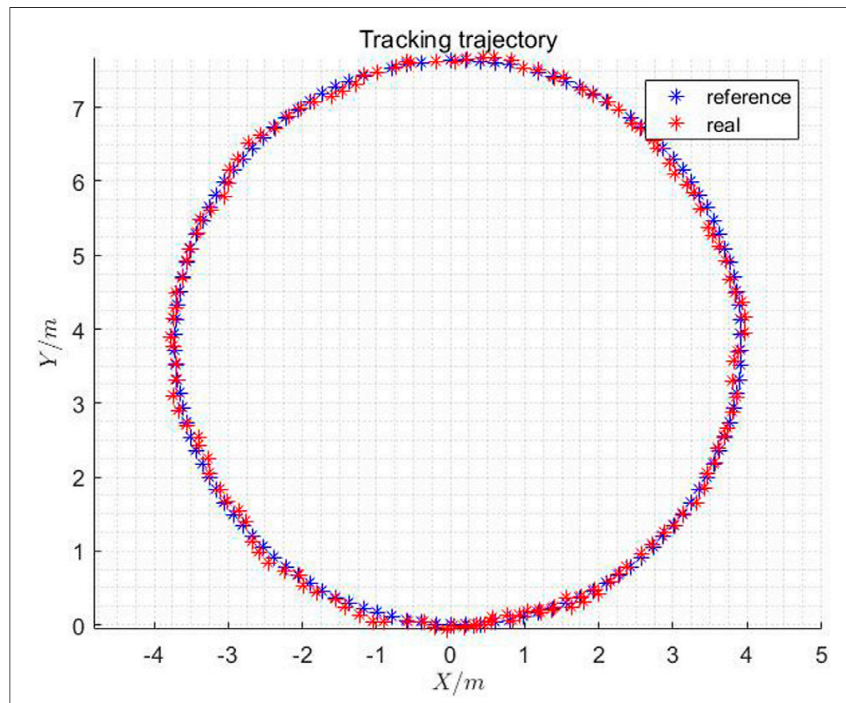

FIGURE 3 | Trajectory of the closed-loop system with the proposed stochastic MPC.

$$
V_{q}=\left[\begin{array}{ccc}
15 & 0 & 0 \\
0 & 22 & 0 \\
0 & 0 & 15 \\
-15 & 0 & 0 \\
0 & -22 & 0 \\
0 & 0 & -15
\end{array}\right], \quad p_{x}=\left[\begin{array}{c}
0.8 \\
0.75 \\
0.7 \\
0.8 \\
0.75 \\
0.7
\end{array}\right]
$$

In the stochastic MPC, the predictive horizon is chosen to be $N=10$; weighting matrices are selected by

$$
Q=\left[\begin{array}{ccc}
30 & 0 & 0 \\
0 & 30 & 0 \\
0 & 0 & 1
\end{array}\right], \quad R=\left[\begin{array}{cc}
0.1 & 0 \\
0 & 0.1
\end{array}\right]
$$

The terminal constraint can be calculated by the learningbased algorithm (42)-(47), and can be illustrated by Figure 2 .

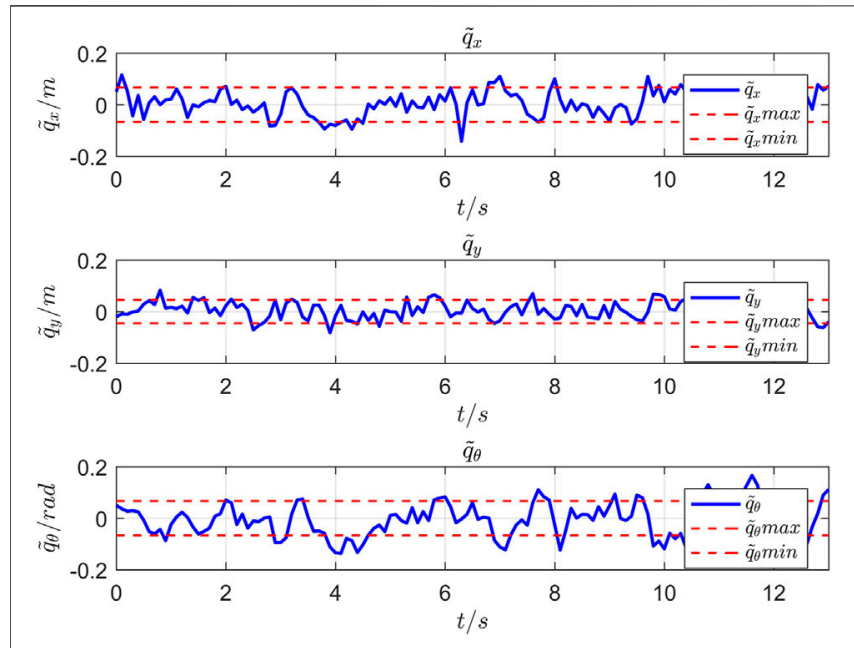

FIGURE 4 | Tracking errors of the closed-loop system with the proposed stochastic MPC.

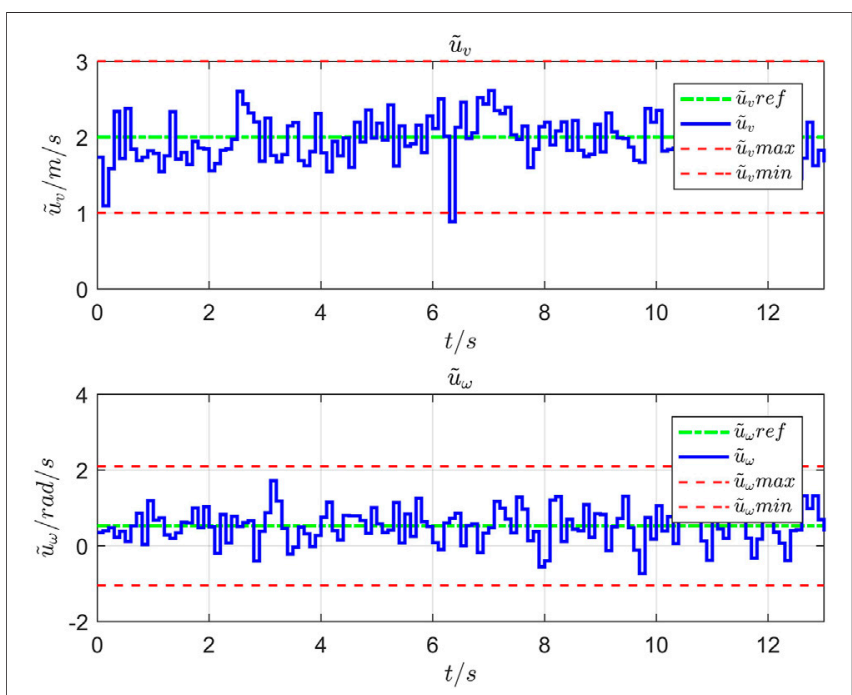

FIGURE 5 | Control inputs of the closed-loop system with the proposed stochastic MPC.

Simulation results are displayed in Figures 3-5. In Figure 3, it can be seen that, with the proposed stochastic MPC, the wheeled mobile robot is capable of tracking its reference trajectory within the task horizon. Only limited tracking errors are witnessed in Figure 4. This simulation example has been run for 500 times with stochastic $\mathbf{w}$, where $\mathbf{p}_{x}$ in (33) can be calculated approximately by

$$
[0.8026,0.7527,0.7047,0.8006,0.7491,0.7008]^{T} \text {, }
$$

and $\mathbf{p}_{x}$ in (6) can be calculated approximately by

$$
[0.8943,0.8539,0.8064,0.8935,0.8512,0.8030]^{T} \text {, }
$$

which is in well accordance with the value given in (60). It can be seen from Figure 5 that the control inputs almost always satisfy 


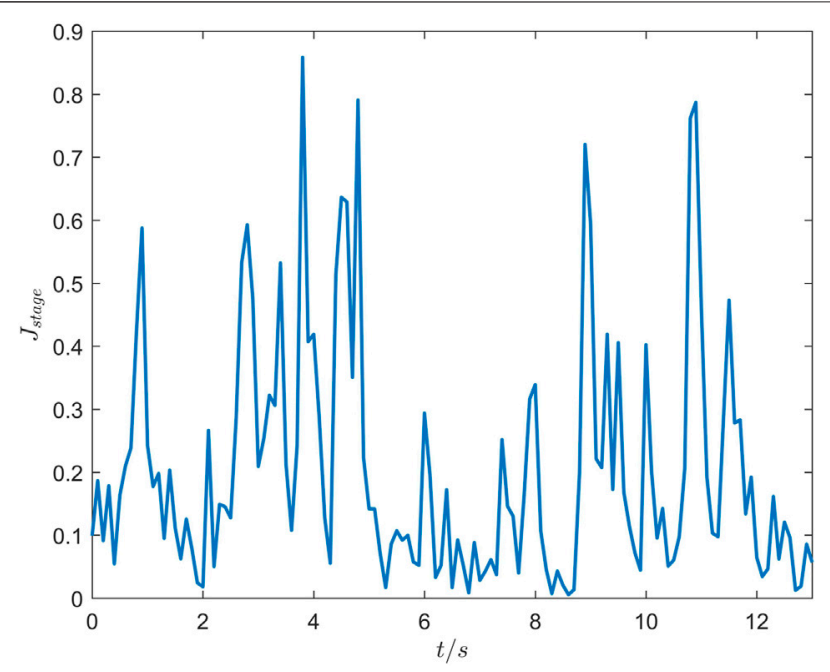

FIGURE 6 | The running stage cost of the closed-loop system.

their constraints, where the constraint on $\tilde{u}_{v}$ has been violated only once, indicating that the probability constraints given in (59) are satisfied. Variation of the running stage cost can be displayed to evaluate the energy efficiency of the closed-loop system. The running state cost is defined by

$$
J_{\text {stage }}(k)=\|\tilde{\mathbf{q}}(k)\|_{Q}^{2}+\|\tilde{\mathbf{u}}(k)\|_{R}^{2},
$$

where the first term implies the accuracy of tracking, and the second term indicates the energy consumption (or the consumed control power to eliminate the tracking error). It can be seen from Figure $\mathbf{6}$ that, in case of stochastic disturbances, the running stage cost can be maintained small. It implies that, the tracking error remains asymptotically average bounded, whereas the energy consumption is small.

\section{REFERENCES}

Alcalá, E., Puig, V., Quevedo, J., and Rosolia, U. (2020). Autonomous Racing Using Linear Parameter Varying-Model Predictive Control (Lpv-mpc). Control. Eng. Pract. 95, 104270. doi:10.1016/j.conengprac.2019.104270

Cairano, S. D., Bernardini, D., Bemporad, A., and Kolmanovsky, I. V. (2014). Stochastic Mpc with Learning for Driver-Predictive Vehicle Control and its Application to Hev Energy Management. IEEE Trans. Contr. Syst. Technol. 22, 1018-1031. doi:10.1109/TCST.2013.2272179

Carrau, J. V., Liniger, A., Zhang, X., and Lygeros, J. (2016). "Efficient Implementation of Randomized Mpc for Miniature Race Cars," in 2016 European Control Conference (ECC), Aalborg, Denmark, June 1-July, 2016, 957-962. doi:10.1109/ECC.2016.7810413

Farina, M., Giulioni, L., and Scattolini, R. (2016). Stochastic Linear Model Predictive Control with Chance Constraints - A Review. J. Process Control. 44, 53-67. doi:10.1016/j.jprocont.2016.03.005

Goncalves, L., Basso, G. F., Dorea, C., and Nascimento, T. P. (2018). "Stochastic Nonlinear Model Predictive mobile Robot Motion Control," in 2018 Latin American Robotic Symposium, 2018 Brazilian Symposium on Robotics (SBR) and 2018 Workshop on Robotics in Education (WRE), Pessoa, Brazil, November 6-10, 2018.

\section{CONCLUSION}

A stochastic MPC is proposed for the wheeled mobile robot subject to probability constraints and stochastic disturbance to track its reference trajectory within the task horizon. The motion of the mobile robot is forecast and optimized by using its linearized discretized model. The cost function in the proposed MPC is designed to penalize its tracking error and energy consumption. By using the tightening probability reachable set technique, the probability constraints can be transformed into deterministic constraints, facilitating the MPC design. The terminal constraint is calculated with a learning-based algorithm. It is proved theoretically that, the optimization is recursively feasible, and the tracking error of the closed-loop system with the proposed stochastic MPC is asymptotically average bounded within the task horizon. The tracking error and statistical data in simulation imply that the performance of the proposed stochastic MPC is satisfactory.

\section{DATA AVAILABILITY STATEMENT}

The raw data supporting the conclusions of this article will be made available by the authors, without undue reservation.

\section{AUTHOR CONTRIBUTIONS}

WZ designed the main algorithm, implemented the simulation example, produced the main results, and wrote the main parts of the paper. BZ initiated the main idea, revised and polished the paper.

\section{FUNDING}

This work was supported by National Natural Science Foundation of China under grant no. 62073015.

Gonzalez, R., Fiacchini, M., Alamo, T., Guzmán, J. L., and Rodríguez, F. (2011) Online Robust Tube-Based Mpc for Time-Varying Systems: A Practical Approach. Int. J. Control. 84, 1157-1170. doi:10.1080/00207179.2011.594093

Hewing, L., Wabersich, K. P., and Zeilinger, M. N. (2020). Recursively Feasible Stochastic Model Predictive Control Using Indirect Feedback. Automatica 119, 109095. doi:10.1016/j.automatica.2020.109095

Hewing, L., and Zeilinger, M. N. (2018). "Stochastic Model Predictive Control for Linear Systems Using Probabilistic Reachable Sets," in 2018 IEEE Conference on Decision and Control (CDC), Miami, FL, December 17-19, 2018, 5182-5188. doi:10.1109/cdc.2018.8619554

Jørgensen, J. B., Sokoler, L. E., Standardi, L., Halvgaard, R., Hovgaard, T. G., Frison, G., et al. (2016). "Economic Mpc for a Linear Stochastic System of Energy Units," in 2016 European Control Conference (ECC), Aalborg, Denmark, June 29-July 1, 2016, 903-909. doi:10.1109/ecc.2016.7810404

Kouvaritakis, B., Cannon, M., Raković, S. V., and Cheng, Q. (2010). Explicit Use of Probabilistic Distributions in Linear Predictive Control. Automatica 46, 1719-1724. doi:10.1016/j.automatica.2010.06.034

Mayne, D. Q., Rawlings, J. B., Rao, C. V., and Scokaert, P. O. M. (2000). Constrained Model Predictive Control: Stability and Optimality. Automatica 36, 789-814. doi:10.1016/s0005-1098(99)00214-9

Mayne, D. (2016). Robust and Stochastic Model Predictive Control: Are We Going in the Right Direction? Annu. Rev. Control. 41, 184-192. doi:10.1016/j.arcontrol.2016.04.006 
Mesbah, A. (2016). Stochastic Model Predictive Control: An Overview and Perspectives for Future Research. IEEE Control. Syst. Mag. 36, 30-44. doi:10.1109/MCS.2016.2602087

Rahmani-andebili, M., and Shen, H. (2017). "Cooperative Distributed Energy Scheduling for Smart Homes Applying Stochastic Model Predictive Control," in 2017 IEEE International Conference on Communications (ICC), Paris, France, May 21-25, 2016, 1-6. doi:10.1109/icc.2017.7996420

Rodas, J., Gonzalez-Prieto, I., Kali, Y., Saad, M., and Doval-Gandoy, J. (2021). Recent Advances in Model Predictive and Sliding Mode Current Control Techniques of Multiphase Induction Machines. Front. Energ. Res. 9, 445. doi:10.3389/fenrg.2021.729034

Scarabaggio, P., Carli, R., Jantzen, J., and Dotoli, M. (2021). "Stochastic Model Predictive Control of Community Energy Storage under High Renewable Penetration," in 2021 29th Mediterranean Conference on Control and Automation (MED), Puglia, Italy, June 22-25, 2021, 973-978. doi:10.1109/MED51440.2021.9480353

Stadler, P., Girardin, L., Ashouri, A., and Maréchal, F. (2018). Contribution of Model Predictive Control in the Integration of Renewable Energy Sources within the Built Environment. Front. Energ. Res. 6, 22. doi:10.3389/ fenrg.2018.00022

Suh, J., Chae, H., and Yi, K. (2018). Stochastic Model-Predictive Control for Lane Change Decision of Automated Driving Vehicles. IEEE Trans. Veh. Technol. 67, 4771-4782. doi:10.1109/TVT.2018.2804891

Sun, Z., Dai, L., Liu, K., Xia, Y., and Johansson, K. H. (2018). Robust Mpc for Tracking Constrained Unicycle Robots with Additive Disturbances. Automatica 90, 172-184. doi:10.1016/j.automatica.2017.12.048

Wang, Q., Duan, Z., Lv, Y., Wang, Q., and Chen, G. (2020). Distributed Model Predictive Control for Linear-Quadratic Performance and Consensus State Optimization of Multiagent Systems. IEEE Trans. Cybern 51, 2905-2915. doi:10.1109/TCYB.2020.3001347

Wu, Z., Rincon, D., and Christofides, P. D. (2020). Process Structure-Based Recurrent Neural Network Modeling for Model Predictive Control of Nonlinear Processes. J. Process Control. 89, 74-84. doi:10.1016/j.jprocont.2020.03.013
Wu, Z., Xia, X., and Zhu, B. (2015). Model Predictive Control for Improving Operational Efficiency of Overhead Cranes. Nonlinear Dyn. 79, 2639-2657. doi:10.1007/s11071-014-1837-8

Yang, W., Xu, D., Jiang, B., and Shi, P. (2021). A Novel Dual-Mode Robust Model Predictive Control Approach via Alternating Optimizations. Automatica 133, 109857. doi:10.1016/j.automatica.2021.109857

Yang, Y., Chen, Y., Tang, C., and Chai, L. (2017). "Quadrotor Helicopters Trajectory Tracking with Stochastic Model Predictive Control," in 2017 Australian and New Zealand Control Conference (ANZCC), Gold Coast, QLD, December 17-20, 2017 (IEEE), 166-171. doi:10.1109/anzcc.2017.8298505

Zhu, B., Guo, K., and Xie, L. (2018). A New Distributed Model Predictive Control for Unconstrained Double-Integrator Multiagent Systems. IEEE Trans. Automat. Contr. 63, 4367-4374. doi:10.1109/tac.2018.2819429

Zhu, B., Zheng, Z., and Xia, X. (2020). Constrained Adaptive Model-Predictive Control for a Class of Discrete-Time Linear Systems with Parametric Uncertainties. IEEE Trans. Automat. Contr. 65, 2223-2229. doi:10.1109/tac.2019.2939659

Conflict of Interest: The authors declare that the research was conducted in the absence of any commercial or financial relationships that could be construed as a potential conflict of interest.

Publisher's Note: All claims expressed in this article are solely those of the authors and do not necessarily represent those of their affiliated organizations, or those of the publisher, the editors and the reviewers. Any product that may be evaluated in this article, or claim that may be made by its manufacturer, is not guaranteed or endorsed by the publisher.

Copyright (C) 2021 Zheng and Zhu. This is an open-access article distributed under the terms of the Creative Commons Attribution License (CC BY). The use, distribution or reproduction in other forums is permitted, provided the original author(s) and the copyright owner(s) are credited and that the original publication in this journal is cited, in accordance with accepted academic practice. No use, distribution or reproduction is permitted which does not comply with these terms. 